\title{
A note on extensions of Pełczyński's decomposition method in Banach spaces
}

\author{
by \\ Elói Medina Galego (São Paulo)
}

\begin{abstract}
Let $X, Y, A$ and $B$ be Banach spaces such that $X$ is isomorphic to $Y \oplus A$ and $Y$ is isomorphic to $X \oplus B$. In 1996, W. T. Gowers solved the Schroeder-Bernstein problem for Banach spaces by showing that $X$ is not necessarily isomorphic to $Y$. In the present paper, we give a necessary and sufficient condition on sextuples $(p, q, r, s, u, v)$ in $\mathbb{N}$ with $p+q \geq 2, r+s \geq 1$ and $u, v \in \mathbb{N}^{*}$ for $X$ to be isomorphic to $Y$ whenever these spaces satisfy the following decomposition scheme:

$$
\left\{\begin{array}{l}
X^{u} \sim X^{p} \oplus Y^{q}, \\
Y^{v} \sim A^{r} \oplus B^{s} .
\end{array}\right.
$$

Namely, $\Omega=(p-u)(s-r-v)-q(r-s)$ is different from zero and $\Omega$ divides $p+q-u$ and $v$. In other words, we obtain an arithmetic characterization of some extensions of the classical Pełczyński decomposition method in Banach spaces. This result leads naturally to several problems closely related to the Schroeder-Bernstein problem.
\end{abstract}

1. Introduction. Let $X$ and $Y$ be Banach spaces. We write $X \sim Y$ if $X$ is isomorphic to $Y$, and $X \not Y$ otherwise. If $n \in \mathbb{N}^{*}=\{1,2,3, \ldots\}$, then $X^{n}$ denotes the sum of $n$ copies of $X, X \oplus \cdots \oplus X$. It will be useful to define $X^{0}=\{0\}$. We recall that $Y$ is isomorphic to a complemented subspace of $X$ if there exists a Banach space $A$ such that $X \sim Y \oplus A$.

Suppose now that $X, Y, A$ and $B$ are Banach spaces satisfying

$$
\left\{\begin{array}{l}
X \sim Y \oplus A, \\
Y \sim X \oplus B .
\end{array}\right.
$$

In 1996, W. T. Gowers [12] solved the so-called Schroeder-Bernstein problem for Banach spaces by showing that $X$ is not necessarily isomorphic to $Y$. Moreover, this first solution was obtained by the construction of a Banach

2000 Mathematics Subject Classification: Primary 46B03, 46B20. problem.

Key words and phrases: Pełczyński's decomposition method, Schroeder-Bernstein 
space $Z$ satisfying

$$
Z \sim Z^{3} \text { and } Z \nsim Z^{2}
$$

Gowers's solution opened two directions of research. The first is to look for new solutions with some particular properties (see [2]-[6], [8], [13]). The second is to ask what additional conditions on $X, Y, A$ and $B$ satisfying (1.1) ensure that $X$ is isomorphic to $Y$ (see [7], [9]-[11]).

Concerning this last direction, it is well known that Pełczyński's decomposition method [1, p. 64], which has played an important role in the isomorphic theory of classical Banach spaces, states that $X \sim Y$ if these spaces satisfy (1.1) and the following decomposition scheme:

$$
\left\{\begin{array}{l}
X \sim X^{2}, \\
Y \sim Y^{2} .
\end{array}\right.
$$

Furthermore, in [10] a sextuple $(p, q, r, s, u, v)$ in $\mathbb{N}$ with $p+q+u \geq 3$, $(p, q) \neq(0,0), r+s+t \geq 3,(r, s) \neq(0,0), u \geq 1$ and $v \geq 1$ was said to be a Schroeder-Bernstein sextuple for Banach spaces (for short, SBs) if $X \sim Y$ whenever the Banach spaces $X$ and $Y$ satisfy (1.1) for some Banach spaces $A$ and $B$ and the following decomposition scheme holds:

$$
\left\{\begin{array}{l}
X^{u} \sim X^{p} \oplus Y^{q}, \\
Y^{v} \sim X^{r} \oplus Y^{s} .
\end{array}\right.
$$

The number $\diamond=(p-u)(s-v)-r q$ was called the discriminant of the sextuple $(p, q, r, s, u, v)$.

We recall the following result on SBs obtained in [10, Corollary 4.2].

TheOREM 1.1. Let $(p, q, r, s, u, v)$ be a sextuple in $\mathbb{N}$ with $p+q+u \geq 3$, $(p, q) \neq(0,0), r+s+v \geq 3,(r, s) \neq(0,0), u=1$ or $v=1$ or $(p, q)=(1,0)$ or $(r, s)=(0,1)$, and with discriminant $\diamond$. Then $(p, q, r, s, u, v)$ is a SBs if and only if $\diamond$ is different from zero and $\diamond$ divides $p+q-u$ and $r+s-v$.

It is an open problem to complete the characterization of the sextuples in $\mathbb{N}$ which are SBs (see [10, Conjecture 4.3 and Problem 4.4]). The present work is a continuation of [10] in the sense that we present new extensions of Pełczyński's decomposition method in Banach spaces. This time, the starting point is the simple fact that from (1.1) we deduce that

$$
Y \sim X \oplus B \sim Y \oplus A \oplus B .
$$

Adding $A \oplus B$ to both sides of (1.5) we have

$$
Y \sim Y \oplus A \oplus B \sim Y \oplus A^{2} \oplus B^{2} .
$$

Therefore by induction we conclude that

$$
Y \sim Y \oplus A^{m} \oplus B^{m}, \quad \forall m \in \mathbb{N} .
$$


So if we want to obtain the second condition of the decomposition scheme (1.3) from (1.1) it suffices to require the strong condition $Y \sim A^{m} \oplus B^{m}$, for some $m \in \mathbb{N}^{*}$. Hence according to Pełczyński's decomposition method $X \sim Y$ whenever there exist Banach spaces $A$ and $B$ satisfying (1.1) and $m \in \mathbb{N}^{*}$ such that the following decomposition scheme holds:

$$
\left\{\begin{array}{l}
X \sim X^{2}, \\
Y \sim A^{m} \oplus B^{m} .
\end{array}\right.
$$

It is then natural to pose, in the spirit of [10], the following problem:

Problem 1.2. Is it possible to describe all decomposition schemes similar to (1.7) which added to (1.1) also yield $X \sim Y$ ?

In order to formulate Problem 1.2 more precisely we introduce the following definition:

Definition 1.3. A sextuple $(p, q, r, s, u, v)$ in $\mathbb{N}$, with $p+q \geq 2, r+s \geq 1$ and $u, v \in \mathbb{N}^{*}$, is a strong Schroeder-Bernstein sextuple for Banach spaces (for short, SSBs) if $X \sim Y$ whenever there exist Banach spaces $A$ and $B$ satisfying (1.1) and the following decomposition scheme:

$$
\left\{\begin{array}{l}
X^{u} \sim X^{p} \oplus Y^{q}, \\
Y^{v} \sim A^{r} \oplus B^{s} .
\end{array}\right.
$$

We also say that $\Omega=(p-u)(s-r-v)-q(r-s)$ is the strong discriminant of the sextuple $(p, q, r, s, u, v)$.

Notice that by $(1.7),(2,0, m, m, 1,1)$ is a SSBs for every $m$ in $\mathbb{N}^{*}$. Furthermore, by using the Banach space $Z$ mentioned in (1.2) we see that $(1,2,2,1,1,1)$ is not a SSBs. Indeed, take $X=Z^{2}, Y=Z$ and $A=B=Z$. According to (1.2), it follows that (1.1) is satisfied, $X \nsim Y$ and also

$$
\left\{\begin{array}{l}
X \sim X \oplus Y^{2}, \\
Y \sim A^{2} \oplus B .
\end{array}\right.
$$

Problem 1.2 asks whether it is possible to determine all SSBs. The main aim of this paper is to solve this problem. Indeed, we shall see that certain families of Banach spaces constructed by W. T. Gowers and B. Maurey in 1997 (see Remark 3.1) are large enough to provide the following characterization of the SSBs in terms of their strong discriminants $\Omega$.

Theorem 1.4. A sextuple $(p, q, r, s, u, v)$ in $\mathbb{N}$ with $p+q \geq 2, r+s \geq 1$ and $u, v \in \mathbb{N}^{*}$ is a SSBs if and only if its strong discriminant $\Omega$ is different from zero and $\Omega$ divides $p+q-u$ and $v$.

The proof of this theorem is quite long and it will be done by proving four propositions: Propositions 2.2, 3.5, 3.6 and 3.7. 
In the last section we also introduce the notion of tight Schroeder-Bernstein sextuples for Banach space and indicate their characterization. We end the paper by posing some problems related to the decomposition scheme (1.8): see Problems 4.3, 4.4 and 4.5.

2. Sufficient condition for a sextuple in $\mathbb{N}$ to be a SSBs. The purpose of this section is to prove Proposition 2.2, which is the sufficiency part of Theorem 1.4.

Proposition 2.2. Let $(p, q, r, s, u, v)$ be a sextuple in $\mathbb{N}$ with $p+q \geq 2$, $r+s \geq 1, u, v \in \mathbb{N}^{*}$ and with strong discriminant $\Omega$. If $\Omega$ is different from zero, and $\Omega$ divides $p+q-u$ and $v$, then $(p, q, r, s, u, v)$ is a SSBs.

Proof. Let $X, Y, A$ and $B$ be Banach spaces satisfying (1.1) and (1.8). We will prove that $X \sim Y$. Let $d=s-r$. Then by the definition of $\Omega$,

$$
\Omega=d(p+q-u)-(p-u) v .
$$

We distinguish five cases: $d=0 ; u=p ; d>0$ and $u>p ; d>0$ and $u<p$; $d<0$.

CASE 1: $d=0$. Then $r=s \geq 1$ and $\Omega=(u-p) v$. Adding $Y$ to both sides of the second condition of (1.8) and using (1.6) with $m=r$, we get

$$
\left\{\begin{array}{l}
Y \sim Y^{v+1}, \\
X^{u} \sim Y^{q} \oplus X^{p} .
\end{array}\right.
$$

Since the discriminant $\diamond$ of the sextuple $(v+1,0, q, p, 1, u)$ is $v(p-u)=-\Omega$, it follows by hypothesis that $\diamond \neq 0$ and $\diamond$ divides $(v+1)-1=v$ and $q+p-u$. So Theorem 1.1 implies that $X \sim Y$.

CASE 2: $u=p$. Then $\Omega=d q \neq 0$ and $d q$ divides $q$. Hence $d=-1$ or $d=1$.

SubCASE 2.1: $d=-1$. Then $r=s+1$. Therefore the second condition of (1.8) implies that

$$
Y^{v} \sim A^{s+1} \oplus B^{s}
$$

Adding $Y$ to both sides of (2.2), by (1.6) with $m=s$ we see that

$$
Y^{v+1} \sim Y \oplus A \sim X .
$$

On the other hand, adding $B^{p}$ to both sides of the first condition of (1.8), by (1.1) we deduce that

$$
Y^{p} \sim(X \oplus B)^{p} \sim X^{p} \oplus B^{p} \sim X^{p} \oplus Y^{q} \oplus B^{p} \sim(X \oplus B)^{p} \oplus Y^{q} \sim Y^{p+q} .
$$

Thus according to $(2.3)$ we conclude that

$$
\left\{\begin{array}{l}
X \sim Y^{v+1} \\
Y^{p} \sim Y^{p+q} .
\end{array}\right.
$$


Since the discriminant $\diamond$ of the sextuple $(0, v+1,0, p+q, 1, p)$ is equal to $-q=\Omega$, we have $\diamond \neq 0$ and $\diamond$ divides $(v+1)-1=v$ and $q$. Again Theorem 1.1 implies that $X \sim Y$.

Subcase 2.2: $d=1$. Then $s=r+1$. By the second condition of (1.8) we know that

$$
Y^{v} \sim A^{r} \oplus B^{r+1} .
$$

Adding $X$ to both sides of (2.4), by the analogue of (1.6) for $X$ with $m=r$ we infer that

$$
Y^{v} \oplus X \sim X \oplus B \sim Y .
$$

Hence again by the first condition of (1.8) we obtain

$$
\left\{\begin{array}{l}
Y \sim Y^{v} \oplus X \\
X^{p} \sim Y^{q} \oplus X^{p} .
\end{array}\right.
$$

Since the discriminant $\diamond$ of the sextuple $(v, 1, q, p, 1, p)$ is $-q=\Omega$, it follows that $\diamond \neq 0$ and $\diamond$ divides $(v+1)-1=v$ and $q$. Once more Theorem 1.1 implies that $X \sim Y$.

Before we consider Cases 3 and 4 note that if $d>0$, then adding $Y$ to both sides of the second condition of (1.8), by (1.6) with $m=r$ we see that

$$
Y^{v+1} \sim Y \oplus B^{d}
$$

Now adding $A^{d}$ to both sides of (2.5), by (1.6) with $m=d$ we get

$$
Y \sim Y^{v+1} \oplus A^{d} .
$$

Next adding $Y \oplus A^{d}$ to both sides of (2.6) we deduce that

$$
Y \sim Y^{v+1} \oplus A^{d} \sim Y^{2 v+1} \oplus A^{2 d} .
$$

Therefore by induction we have

$$
Y \sim Y^{i v+1} \oplus A^{i d}, \quad \forall i \in \mathbb{N}^{*} .
$$

CASE 3: $d>0$ and $u>p$. In this case, $u \geq p+q$. Otherwise, $u<p+q$ and by (2.1), $\Omega>v$, which is absurd, because by hypothesis $\Omega$ divides $v$. There are two subcases: $\Omega>0$ and $\Omega<0$.

SubCase 3.1: $\Omega>0$. Let $m, n \in \mathbb{N}$ be such that $p+q-u=-m \Omega$ and $v=n \Omega$.

Adding $B^{p}$ to both sides of the first condition of (1.8) we conclude that $Y^{p+q} \sim(X \oplus B)^{p} \oplus Y^{q} \sim X^{u-p} \oplus X^{p} \oplus B^{p} \sim(Y \oplus A)^{u-p} \oplus Y^{p} \sim Y^{u} \oplus A^{u-p}$. Consequently,

$$
Y^{n(p+q)} \sim Y^{n u} \oplus A^{n(u-p)} .
$$


Fix $j \in \mathbb{N}^{*}$ such that $j v+1>n(p+q)$. By (2.7) with $i=j$ and (2.8) we have

$$
\begin{aligned}
Y & \sim Y^{j v+1-n(p+q)} \oplus Y^{n u} \oplus A^{n(u-p)} \oplus A^{j d} \\
& \sim Y^{j v+1-n(p+q-u)} \oplus A^{j d+n(u-p)} .
\end{aligned}
$$

Moreover, by (2.7) with $i=j+m$, it follows that

$$
Y \sim Y^{j v+1+m v} \oplus A^{j d+m d} .
$$

By (2.1) and the choice of $m$ and $n$ we obtain $-n(p+q-u)=m v$ and $1+m d=n(u-p)$. Hence, by using (2.10) in (2.9) we see that $Y \sim Y \oplus A \sim X$.

SubCaSe 3.2: $\Omega<0$. Pick $m, n \in \mathbb{N}$ such that $p+q-u=m \Omega$ and $v=-n \Omega$. Proceeding as in Subcase 3.1 we get (2.9) and (2.10). Observe that $-n(p+q-u)=m v$ and $1+n(u-p)=m d$. Thus by using (2.9) in (2.10) we deduce that $Y \sim Y \oplus A \sim X$.

CASE 4: $d>0$ and $u<p$. There are two subcases: $\Omega>0$ and $\Omega<0$.

SubCASE 4.1: $\Omega>0$. Let $m, n \in \mathbb{N}$ be such that $p+q-u=m \Omega$ and $v=n \Omega$. Adding $A^{u}$ to both sides of the first condition of (1.8) we infer that $Y^{u} \sim X^{u} \oplus A^{u} \sim X^{p-u} \oplus X^{u} \oplus A^{u} \oplus Y^{q} \sim(Y \oplus A)^{p-u} \oplus Y^{u} \oplus Y^{q} \sim Y^{p+q} \oplus A^{p-u}$.

Consequently,

$$
Y^{n u} \sim Y^{n(p+q)} \oplus A^{n(p-u)} .
$$

Fix $j \in \mathbb{N}^{*}$ such that $j v+1>n u$. By (2.7) with $i=j$ and (2.11) we have

$$
\begin{aligned}
Y & \sim Y^{j v+1-n u} \oplus Y^{n(p+q)} \oplus A^{n(p-u)} \oplus A^{j d} \\
& \sim Y^{j v+1+n(p+q-u)} \oplus A^{j d+n(p-u)} .
\end{aligned}
$$

Furthermore, by (2.7) with $i=j+m$, it follows that

$$
Y \sim Y^{j v+1+m v} \oplus A^{j d+m d} .
$$

Now notice that $n(p+q-u)=m v$ and $1+n(p-u)=m d$. Hence by using (2.12) in (2.13) we deduce that $Y \sim Y \oplus A \sim X$.

SUBCASE 4.2: $\Omega<0$. Let $m, n \in \mathbb{N}$ be such that $p+q-u=-m \Omega$ and $v=-n \Omega$. Similarly to Subcase 4.1 we obtain (2.12) and (2.13). Note that $n(p+q-u)=m v$ and $1+m d=n(p-u)$. Therefore by using (2.13) in (2.12) we obtain $Y \sim Y \oplus A \sim X$.

CASE 5: $d<0$. In this case observe that if $u>p$ and $u>p+q$, then by (2.1), $\Omega>v$, which is absurd. Further, if $u<p$, then again by (2.1), $\Omega<-v$, which is also absurd. So $u>p$ and $u \leq p+q$. There are two subcases: $\Omega>0$ and $\Omega<0$. 
SubCASE 5.1: $\Omega>0$. Let $m, n \in \mathbb{N}$ be such that $p+q-u=m \Omega$ and $v=n \Omega$. From the first condition of (1.8) and (1.1) we deduce that

$$
X^{u} \sim X^{p} \oplus(X \oplus B)^{q} \sim X^{p+q} \oplus B^{q} .
$$

Consequently,

$$
X^{n u} \sim X^{(n(p+q)} \oplus B^{n q} .
$$

On the other hand, adding $Y$ to both sides of the second condition of (1.8), by (1.6) with $m=s$ we see that

$$
Y^{v+1} \sim Y \oplus A^{-d}
$$

Adding $B^{-d}$ to both sides of (2.15), by (1.6) with $m=-d$ we infer

$$
Y \sim Y^{v+1} \oplus B^{-d} \text {. }
$$

Then we have

$$
X \oplus B \sim Y \sim Y^{v+1} \oplus B^{-d} \sim(X \oplus B)^{v+1} \oplus B^{-d} \sim X^{v+1} \oplus B^{-d+v+1} .
$$

Adding $A$ to both sides of (2.16), by the analogue of (1.6) for $X$ with $m=1$ we conclude that

$$
X \sim X^{v+1} \oplus B^{-d+v}
$$

Next adding $X^{v} \oplus B^{-d+v}$ to both sides of (2.17) we have

$$
X \sim X^{v+1} \oplus B^{-d+v} \sim X^{2 v+1} \oplus B^{2(-d+v)} .
$$

Therefore by induction we get

$$
X \sim X^{i v+1} \oplus B^{i(-d+v)}, \quad \forall i \in \mathbb{N}^{*} .
$$

Pick $j \in \mathbb{N}^{*}$ such that $j v+1>n u$. By (2.18) with $i=j$ and (2.14) we obtain

$$
\begin{aligned}
X & \sim Y^{j v+1-n u} \oplus X^{n(p+q)} \oplus B^{n q} \oplus B^{j(-d+v)} \\
& \sim X^{j v+1+n(p+q-u)} \oplus B^{j(-d+v)+n q} .
\end{aligned}
$$

Moreover, by (2.18) with $i=j+m$, it follows that

$$
X \sim X^{j v+1+m v} \oplus B^{j(-d+v)+m(-d+v)} .
$$

Notice that $n(p+q-u)=m v$ and $1=m d+n(u-p)$. So $n q=m v+n(u-p)=$ $m v+(1-m d)=m(-d+v)+1$. Hence by using (2.19) in (2.20), it follows that $X \sim X \oplus B \sim Y$.

SubCASE 5.2: $\Omega<0$. Let $m, n \in \mathbb{N}$ be such that $p+q-u=-m \Omega$ and $v=-n \Omega$. Proceeding as in Subcase 5.1 we obtain (2.19) and (2.20). Note that $n(p+q-u)=m v$ and $1=-m d-n(u-p)$. Thus $n q=m v+n(u-p)=$ $m v-m d-1=m(-d+v)$. Therefore by using (2.20) in (2.19) we see that $X \sim X \oplus B \sim Y$.

3. Necessary condition for a sextuple in $\mathbb{N}$ to be a SSBs. Our task in this section is to prove the necessity part of Theorem 1.4. In order 
to do this we prove Propositions 3.5, 3.6 and 3.7. We start by recalling some Banach spaces introduced by W. T. Gowers and B. Maurey in 1997.

REMARK 3.1. In [13, p. 563] Banach spaces $X_{t}$ were constructed, for every $t \in \mathbb{N}, t \geq 2$, having the following property: $X_{t}^{m} \sim X_{t}^{n}$, with $m, n \in \mathbb{N}^{*}$, if and only if $m \equiv n \bmod t$.

In order to prove our propositions we need three lemmas. They relate to the Banach spaces $X_{t}$ mentioned in Remark 3.1.

Lemma 3.2. Let $p, q, r, s, u, v \in \mathbb{N}$ with $p+q \geq 2, r+s \geq 1$ and $u, v \in \mathbb{N}^{*}$ and suppose that there exist $i, j, t \in \mathbb{N}^{*}$ with $t \geq 2$ satisfying

(a) $t$ divides $i(p-u)+j q$;

(b) $t$ divides $i(r-s)+j(s-r-v)$;

(c) $t$ does not divide $j-i$.

Then $(p, q, r, s, u, v)$ is not a SSBs.

Proof. Let $n \in \mathbb{N}^{*}$ be such that $n t-j+i>0$ and $n t-i+j>0$. Since $j+(n t-j+i)-i=n t$ and $i+(n t-i+j)-j=n t$, by the property of $X_{u}$ mentioned in Remark 3.1 we have

$$
\left\{\begin{array}{l}
X_{t}^{i} \sim X_{t}^{j} \oplus X_{t}^{n t-j+i} \\
X_{t}^{j} \sim X_{t}^{i} \oplus X_{t}^{n t-i+j}
\end{array}\right.
$$

From conditions (a) and (b) we deduce that

$$
\left\{\begin{array}{l}
X_{t}^{i u} \sim X_{t}^{i p} \oplus X_{t}^{j q}, \\
X_{t}^{j v} \sim X_{t}^{(n t-j+i) r} \oplus X_{t}^{(n t-i+j) s} .
\end{array}\right.
$$

Further according to condition (c) we conclude that $X_{t}^{i}$ is not isomorphic to $X_{t}^{j}$. Consequently, $(p, q, r, s, u, v)$ is not a SSBs.

Lemma 3.3. Let $p, q, r, s, u, v \in \mathbb{N}$ with $p+q \geq 2, r+s \geq 1$ and $u, v \in \mathbb{N}^{*}$ and with strong discriminant $\Omega \geq 2$. Suppose that there exist integers $\alpha$ and $\beta$ satisfying

(a) $\alpha(s-r-v)>\beta q$;

(b) $\beta(p-u)>\alpha(r-s)$;

(c) $\Omega$ does not divide $\beta(p+q-u)+\alpha v$.

Then $(p, q, r, s, u, v)$ is not a SSBs.

Proof. Let $t=\Omega$ and consider the linear system

$$
\left\{\begin{array}{l}
i(p-u)+j q=\alpha t, \\
i(r-s)+j(s-r-v)=\beta t .
\end{array}\right.
$$

The only solution of (3.1) is $i=\alpha(s-r-v)-\beta q$ and $j=\beta(p-u)+\alpha(r-s)$. It follows from (a) $-(\mathrm{c})$ that $i, j>0$ and $t$ does not divide $j-i=\beta(p+q-u)+\alpha v$. 
Moreover, clearly $t$ divides $i(p-u)+j q$ and $i(r-s)+j(s-r-v)$. Therefore Lemma 3.2 implies that $(p, q, r, s, u, v)$ is not a SSBs.

Taking $t=-\Omega$ and proceeding as in the proof of Lemma 3.3 we obtain:

Lemma 3.4. Let $p, q, r, s, u, v \in \mathbb{N}$ with $p+q \geq 2, r+s \geq 1, u, v, t \in \mathbb{N}^{*}$ and with strong discriminant $\Omega \leq-2$. Suppose that there exist integers $\alpha$ and $\beta$ satisfying

(a) $\alpha(s-r-v)<\beta q$;

(b) $\beta(p-u)<\alpha(r-s)$;

(c) $\Omega$ does not divide $\beta(p+q-u)+\alpha v$.

Then $(p, q, r, s, u, v)$ is not a SSBs.

Proposition 3.5. If a sextuple $(p, q, r, s, u, v)$ in $\mathbb{N}$ with $p+q \geq 2$, $r+s \geq 1$ and $u, v \in \mathbb{N}^{*}$ is a SSBs, then its strong discriminant $\Omega$ is different from zero.

Proof. Suppose that $\Omega=0$. We distinguish four cases: $q=0 ; p=u$ and $q>0 ; p>u$ and $q>0 ; p<u$ and $q>0$.

CASE 1: $q=0$. Then $p \geq 2$ and since $\Omega=(p-u)(s-r-v)=0$, we get $p=u$ or $s=r+v$.

SubCASE 1.1: $p=u$. Take $n \in \mathbb{N}^{*}$ such that $-n v=v+r-s-2, i=n$, $j=n+1$ and $t \in \mathbb{N}$ satisfying $-t=n(r-s)+(n+1)(s-r-v)$. Thus $t \geq 2$, $t$ divides $i(r-s)+j(s-r-v)$ and $t$ does not divide $j-i=1$. So Lemma 3.2 implies that $(p, q, r, s, u, v)$ is not a SSBs.

SubCASE 1.2: $s=r+v$. By Remark 2.1 we have

$$
\left\{\begin{array} { l } 
{ X _ { 2 } ^ { 2 } \sim X _ { 2 } \oplus X _ { 2 } , } \\
{ X _ { 2 } \sim X _ { 2 } ^ { 2 } \oplus X _ { 2 } , }
\end{array} \quad \left\{\begin{array}{l}
X_{2}^{2 v} \sim X_{2}^{2 p} \\
X_{2}^{v} \sim X_{2}^{r} \oplus X_{2}^{r+v} .
\end{array}\right.\right.
$$

Since $X_{2}^{2} \not X_{2}$, it follows that $(p, q, r, s, u, v)$ is not a SSBs.

CASE 2: $p=u$ and $q>0$. Hence $\Omega=-q(r-s)=0$ and $r=s$. Thus by Remark 3.1 we see that

$$
\left\{\begin{array} { l } 
{ X _ { 2 } \sim X _ { 2 } ^ { 2 } \oplus X _ { 2 } , } \\
{ X _ { 2 } ^ { 2 } \sim X _ { 2 } \oplus X _ { 2 } , }
\end{array} \quad \left\{\begin{array}{l}
X_{2}^{p} \sim X_{2}^{p} \oplus X_{2}^{2 q}, \\
X_{2}^{2 v} \sim X_{2}^{r} \oplus X_{2}^{r} .
\end{array}\right.\right.
$$

Consequently, $(p, q, r, s, u, v)$ is not a SSBs.

CASE 3: $p>u$ and $q>0$. Take $i=p$ and $j=p-u$. So $i(p-u)+j q=$ $(p-u)(p+q)$ and since $\Omega=0$, it follows that $i(r-s)+j(s-r-u)=$ $(r-s)(p+q)$. Furthermore, $j-i=-u$ and $p+q$ does not divide $u$. Thus it suffices to take $t=p+q$ and apply Lemma 3.2 to see that $(p, q, r, s, u, v)$ is not a SSBs. 
CASE 4: $p<u$ and $q>0$. Take $i=q$ and $j=u-p$. Therefore $i(p-u)+$ $j q=0$ and since $\Omega=0$, we have $i(r-s)+j(s-r-u)=0$. Moreover $u \neq p+q$. Otherwise from $\Omega=(p-u)(s-r-v)-q(r-s)=0$ we would conclude that $q v=0$, which is absurd. Now take $t \in \mathbb{N}, t \geq 2$, such that $t$ does not divide $j-i=u-p-q$ and apply Lemma 2.2 to deduce that $(p, q, r, s, u, v)$ is not a SSBs.

Proposition 3.6. If a sextuple $(p, q, r, s, u, v)$ in $\mathbb{N}$ with $p+q \geq 2$, $r+s \geq 1, u, v \in \mathbb{N}^{*}$ and with strong discriminant $\Omega$ different from zero is a SSBs, then $\Omega$ divides $p+q-u$.

Proof. Assume that $\Omega \neq 0$ and $\Omega$ does not divide $p+q-u$. We consider two cases: $\Omega \geq 2$ and $\Omega \leq-2$.

CASE 1: $\Omega \geq 2$. We distinguish four subcases: $q=0 ; p=u$ and $q>0$; $p>u$ and $q>0 ; p<u$ and $q>0$.

Subcase 1.1: $q=0$. Then $\Omega=(p-u)(s-r-v)$ and hence $p \neq u$. Note that in this subcase $(p, 0, r, s, u, v)$ is a SSBs if and only if $(u, 0, r, s, p, v)$ is. Thus we can suppose $p>u$ and therefore $s-r-v>0$. Take $\alpha=p-u$ and $\beta=r-s+1$. Since $(p-u)(r-s)<(p-u)(r-s+1)$ and $\beta(p+q-u)+\alpha v=$ $(p-u)(r-s+v)+p-u$, it is enough to apply Lemma 3.3 to see that $(p, q, r, s, u, v)$ is not a SSBs.

SubCASE 1.2: $p=u$ and $q>0$. Then $\Omega=-q(r-s)$. Take $\alpha=q$ and $\beta=s-r-v-1$. Since $q(s-r-v-1)<q(s-r-v)$ and $\beta q+\alpha v=(s-r) q-q$, Lemma 3.3 implies that $(p, q, r, s, u, v)$ is not a SSBs.

SubCASE 1.3: $p>u$ and $q>0$. By $(2.1),(r-s) /(p-u)<(s-r-v) / q$. Let $m \in \mathbb{N}^{*}$ be such that

$$
\frac{r-s}{p-u}<\frac{s-r-v}{q}-\frac{1}{q m}
$$

and take $\alpha=q m$ and $\beta=m(s-r-v)-1$. Therefore $(r-s) /(p-u)<$ $\beta / \alpha<(s-r-v) / q$ and $\beta(p+q-u)+\alpha v=m \Omega-(p+q-u)$. Again by Lemma 3.3, $(p, q, r, s, u, v)$ is not a SSBs.

SubCase 1.4: $p<u$ and $q>0$. By $(2.1),(s-r-v) / q<(r-s) /(p-u)$. Fix $m \in \mathbb{N}^{*}$ such that

$$
\frac{s-r-v}{q}+\frac{1}{q m}<\frac{r-s}{p-u},
$$

and take $\alpha=q m$ and $\beta=m(s-r-v)+1$. Hence $(s-r-v) / q<\beta / \alpha<$ $(r-s) /(p-u)$ and $\beta(p+q-u)+\alpha v=m \Omega+(p+q-u)$. Once more according to Lemma 3.3, $(p, q, r, s, u, v)$ is not a SSBs.

CASE 2: $\Omega \leq-2$. We distinguish four subcases: $q=0 ; p=v$ and $q>0$; $p>v$ and $q>0 ; p<v$ and $q>0$. 
Subcase 2.1: $q=0$. Then $\Omega=(p-u)(s-r-v)$ and as in Subcase 1.1 we may assume that $p>u$. So $s-r-v<0$. Take $\alpha=p-u$ and $\beta=r-s-1$. Since $(p-u)(r-s-v),(p-u)(r-s)$ and $\beta(p-u)+\alpha v=(r-s+v)(p-u)-(p-u)$, Lemma 3.3 shows that $(p, q, r, s, u, v)$ is not a SSBs.

SuBCASE 2.2: $p=u$ and $q>0$. Then $\Omega=-q(r-s)$. Take $\alpha=q$ and $\beta=s-r-v+1$. Since $q(s-r-v)<q(s-r-v+1)$ and $\beta q+\alpha v=(s-r) q+q$, it follows from Lemma 3.3 that $(p, q, r, s, u, v)$ is not a SSBs.

SubCASE 2.3: $p>u$ and $q>0$. By $(2.1),(s-r-v) / q<(r-s) /(p-u)$. Pick $m \in \mathbb{N}^{*}$ such that

$$
\frac{s-r-v}{q}+\frac{1}{q m}<\frac{r-s}{p-u},
$$

and take $\alpha=q m$ and $\beta=m(s-r-v)+1$. Therefore $(s-r-v) / q<\beta / \alpha<$ $(r-s) /(p-u)$ and $\beta(p+q-u)+\alpha v=m \Omega+(p+q-u)$. Consequently, Lemma 3.3 implies that $(p, q, r, s, u, v)$ is not a SSBs.

SUBCASE 2.4: $p<u$ and $q>0$. By $(2.1),(r-s) /(p-u)<(s-r-v) / q$. Let $m \in \mathbb{N}^{*}$ be such that

$$
\frac{r-s}{p-u}<\frac{s-r-v}{q}-\frac{1}{q m}
$$

and take $\alpha=q m$ and $\beta=m(s-r-v)-1$. Thus $(r-s) /(p-u)<\beta / \alpha<$ $(s-r-v) / q$ and $\beta(p+q-u)+\alpha v=m \Omega-(p+q-u)$. It suffices to apply Lemma 3.3 to see that $(p, q, r, s, u, v)$ is not a SSBs and complete the proof of the proposition.

Proposition 3.7. If a sextuple $(p, q, r, s, u, v)$ in $\mathbb{N}$ with $p+q \geq 2$, $r+s \geq 1, u, v \in \mathbb{N}^{*}$ and strong discriminant $\Omega$ different fom zero is a SSBs, then $\Omega$ divides $v$.

Proof. Assume that $\Omega \neq 0$ and $\Omega$ does not divide $v$. We also consider two cases: $\Omega \geq 2$ and $\Omega \leq-2$.

CASE 1: $\Omega \geq 2$. We distinguish five subcases: $r=s ; s=r+v ; r>s$; $r<s$ and $s<r+v ; r<s$ and $s>r+v$.

Subcase 1.1: $r=s$. Then $\Omega=-(p-u) v$ and $p<u$. Take $\alpha=1-q$ and $\beta=-v$. Since $-q v<(-q+1) v$ and $\beta(p+q-u)+\alpha v=-v(p-u)+v$, Lemma 3.4 implies that $(p, q, r, s, u, v)$ is not a SSBs.

Subcase 1.2: $s=r+v$. Then $\Omega=-q(r-s)$ and $r-s<0$. Take $\alpha=u+1-p$ and $\beta=s-r$. Since $(p-u-1)(r-s)>(p-u)(r-s)$ and $\beta q+\alpha v=q v+v$, Lemma 3.4 shows that $(p, q, r, s, u, v)$ is not a SSBs.

Subcase 1.3: $r>s$. Then $s<r+v$ and by $(2.1),(p-u) /(r-s)<$ $q /(s-r-v)$. Let $m \in \mathbb{N}^{*}$ be such that

$$
\frac{p-u}{r-s}<\frac{q}{s-r-v}+\frac{1}{m(s-r-v)},
$$


and take $\alpha=q m-1$ and $\beta=m(s-r-u)$. Hence $q /(s-r-v)<\alpha / \beta<$ $(p-u) /(r-s)$, and $\beta(p+q-v)+\alpha v=m \Omega+v$. Consequently, Lemma 3.3 implies that $(p, q, r, s, u, v)$ is not a SSBs.

SubCASE 1.4: $r<s$ and $s<r+v$. By $(2.1), q /(s-r-v)<(p-v) /(r-s)$. Fix $m \in \mathbb{N}^{*}$ such that

$$
\frac{q}{s-r-u}-\frac{1}{m(s-r-u)}<\frac{p-v}{r-s},
$$

and take $\alpha=q m+1$ and $\beta=m(s-r-u)$. Note that $q /(s-r-v)<\alpha / \beta<$ $(p-v) /(r-s)$, and $\beta(p+q-v)+\alpha t=m[(p-v)(s-r-u)-(r-s)]-v$. Again by Lemma 3.3, $(p, q, r, s, u, v)$ is not a SSBs.

SubCASE 1.5: $r<s$ and $s>r+v$. By $(2.1),(p-u) /(r-s)<q /(s-r-v)$. Pick $m \in \mathbb{N}^{*}$ such that

$$
\frac{p-u}{r-s}<\frac{q}{s-r-v}-\frac{1}{m(s-r-v)},
$$

and take $\alpha=q m-1$ and $\beta=m(s-r-u)$. Observe that $q /(s-r-v)<$ $\alpha / \beta<(p-u) /(r-s)$, and $\beta(p+q-v)+\alpha v=m \Omega-v$. Once again Lemma 3.3 implies that $(p, q, r, s, u, v)$ is not a SSBs.

CASE 2: $\Omega \leq 2$. We distinguish five subcases: $r=s ; s=r+v ; r>s$; $r<s$ and $s<r+v ; r<s$ and $s>r+v$.

Subcase 2.1: $r=s$. Then $\Omega=-(p-u) v$ and $p>u$. Take $\alpha=q+1$ and $\beta=-v$. Since $(-q-1) v<-q v$ and $\beta(p+q-u)+\alpha v=-v(p-u)+v$, by Lemma 3.4, $(p, q, r, s, u, v)$ is not a SSBs.

Subcase 2.2: $s=r+v$. Then $\Omega=-q(r-s)$ and $r-s>0$. Take $\alpha=p-u+1$ and $\beta=r-s$. Since $(p-u)(r-s)<(p-u+1)(r-s)$ and $\beta q+\alpha v=-q v+v$, Lemma 3.4 implies that $(p, q, r, s, u, v)$ is not a SSBs.

Subcase 2.3: $r>s$. Then $s<r+v$ and by $(2.1), q /(s-r-v)<$ $(p-u) /(r-s)$. Let $m \in \mathbb{N}^{*}$ be such that

$$
\frac{q}{s-r-v}-\frac{1}{m(s-r-v)}<\frac{p-u}{r-s},
$$

and take $\alpha=q m-1$ and $\beta=m(s-r-v)$. Notice that $q /(s-r-v)<$ $\alpha / \beta<(p-u) /(r-s$, and $\beta(p+q-u)+\alpha v=-m \Omega-v$. Consequently, Lemma 3.4 implies that $(p, q, r, s, u, v)$ is not a SSBs.

SubCASE 2.4: $r<s$ and $s<r+v$. By $(2.1),(p-u) /(r-s)<q /(s-r-v)$. Pick $m \in \mathbb{N}^{*}$ such that

$$
\frac{p-u}{r-s}<\frac{q}{s-r-v}+\frac{1}{m(s-r-v)},
$$


and take $\alpha=q m-1$ and $\beta=m(s-r-u)$. Thus $q /(s-r-v)<\alpha / \beta<$ $(p-u) /(r-s)$, and $\beta(p+q-v)+\alpha v=m \Omega+v$. Again by Lemma 3.3, $(p, q, r, s, u, v)$ is not a SSBs.

SUBCASE 2.5: $r<s$ and $s>r+v$. By $(2.1), q /(s-r-v)<(p-u) /(r-s)$. Fix $m \in \mathbb{N}^{*}$ such that

$$
\frac{q}{s-r-v}<\frac{1}{m(s-r-v)}+\frac{p-u}{m(r-s)},
$$

and take $\alpha=q m+1$ and $\beta=m(s-r-u)$. Therefore $q /(s-r-v)<\alpha / \beta<$ $(p-u) /(r-s)$, and $\beta(p+q-v)+\alpha v=m \Omega+v$. Once again Lemma 3.4 implies that $(p, q, r, s, u, v)$ is not a SSBs, and the proposition is proved.

4. Some remarks and problems. Taking into account the analogue of (1.6) for $X$ we are led to

Definition 4.1. A sextuple $(p, q, r, s, u, v)$ in $\mathbb{N}$ with $p+q \geq 1, r+s \geq 1$ and $u, v \in \mathbb{N}^{*}$ is a tight Schroeder-Bernstein sextuple for Banach spaces (for short, TSBs) if $X \sim Y$ whenever there exist Banach spaces $A$ and $B$ satisfying (1.1) and the following decomposition scheme:

$$
\left\{\begin{array}{l}
X^{u} \sim A^{p} \oplus B^{q} \\
Y^{v} \sim A^{r} \oplus B^{s} .
\end{array}\right.
$$

We also say that $\Theta=(p-q-u)(s-r-v)-(q-p)(r-s)$ is the tight discriminant of the sextuple $(p, q, r, s, u, v)$.

Similarly to Theorem 1.4, we can prove:

TheOREM 4.2. A sextuple $(p, q, r, s, u, v)$ in $\mathbb{N}$ with $p+q \geq 1, r+s \geq 1$ and $u, v \in \mathbb{N}^{*}$ is a TSBs if and only its tight discriminant $\Theta$ is different from zero and $\Theta$ divides $u$ and $v$.

This result was proved in [11, Theorem 1.3] for the case $u=v=1$.

However, note that if we replace $B$ by $X$ or $A$ by $Y$ or still $Y$ by $A$ in the second condition of (1.8) some problems arise naturally, for example:

Problem 4.3. Give non-isomorphic Banach spaces $X$ and $Y$ such that there exist Banach spaces $A$ and $B$ satisfying (1.1) and

$$
\left\{\begin{array}{l}
X^{2} \sim X \oplus Y \\
Y \sim A \oplus X
\end{array}\right.
$$

Problem 4.4. Give non-isomorphic Banach spaces $X$ and $Y$ such that there exist Banach spaces $A$ and $B$ satisfying (1.1) and

$$
\left\{\begin{array}{l}
X^{2} \sim X \oplus Y \\
Y \sim Y \oplus B^{2}
\end{array}\right.
$$


Problem 4.5. Give non-isomorphic Banach spaces $X$ and $Y$ such that there exist Banach spaces $A$ and $B$ satisfying (1.1) and

$$
\left\{\begin{array}{l}
X^{2} \sim X \oplus Y, \\
A^{2} \sim A \oplus B .
\end{array}\right.
$$

\section{References}

[1] P. G. Casazza, The Schroeder-Bernstein property for Banach spaces, in: Banach Space Theory (Iowa City, IA, 1987), Contemp. Math. 85, Amer. Math. Soc., 1989, $61-77$.

[2] V. Ferenczi and E. M. Galego, Some results about the Schroeder-Bernstein property for separable Banach spaces, Canad. J. Math. 59 (2007), 63-84.

[3] E. M. Galego, On solutions to the Schroeder-Bernstein problem for Banach spaces, Arch. Math. (Basel) 79 (2002), 299-307.

[4] -, Banach spaces complemented in each other without isomorphic finite sums, Bull. Polish Acad. Sci. Math. 50 (2002), 1-9.

[5] -, The Schroeder-Bernstein index for Banach spaces, Studia Math. 164 (2004), $29-38$.

[6] - On pairs of Banach spaces which are isomorphic to complemented subspaces of each other, Colloq. Math. 101 (2004), 279-287.

[7] -, An arithmetic characterization of decomposition methods in Banach spaces similar to Pełczyński's decomposition method, Bull. Polish Acad. Sci. Math. 52 (2004), $273-282$.

[8] -, A remark on non-separable solutions to the Schroeder-Bernstein problem for Banach spaces, Results Math. 47 (2005), 55-60.

[9] -, An arithmetical characterization of decomposition methods in Banach spaces via supplemented subspaces, Glasgow Math. J. 47 (2005), 489-500.

[10] -, Schroeder-Bernstein quintuples for Banach spaces, Bull. Polish Acad. Sci. Math. 54 (2006), 113-124.

[11] -, On decomposition methods in Banach spaces via supplemented subspaces resembling Petczyński's decomposition methods, Results Math., to appear.

[12] W. T. Gowers, A solution to the Schroeder-Bernstein problem for Banach spaces, Bull. London Math. Soc. 28 (1996), 297-304.

[13] W. T. Gowers and B. Maurey, Banach spaces with small spaces of operators, Math. Ann. 307 (1997), 543-568.

Department of Mathematics - IME

University of São Paulo

São Paulo 05315-970, Brazil

E-mail: eloi@ime.usp.br

Received November 7, 2005

Revised version February 27, 2007 